\title{
„Digitale Kluft: ein zweifelhaftes Konzept
}

Daniel Pimienta

\section{(2) OpenEdition \\ 1 Journals}

Electronic version

URL: http://journals.openedition.org/sjep/520

DOI: $10.4000 /$ sjep.520

ISSN: 1663-9677

Publisher

Institut de hautes études internationales et du développement

\section{Printed version}

Date of publication: 1 novembre 2003

Number of pages: 29-31

ISSN: 1660-5926

\section{Electronic reference}

Daniel Pimienta, « „Digitale Kluft: ein zweifelhaftes Konzept », Schweizerisches Jahrbuch für 520 


\title{
„Digitale Kluft: ein zweifelhaftes Konzept
}

\author{
Daniel Pimienta*
}

D as Konzept der „digitalen Kluft" wird im vorherrschenden - vereinfachenden und oberflächlichen - Diskurs inflationär verwendet. Dieser Diskurs tendiert dazu, die wahren gesellschaftlichen Herausforderungen auszublenden (gesellschaftliche Aneignung der Technologien und damit einhergehend das individuelle und kollektive Empowerment der Menschen) und die Frage des Zugangs - im engen Sinne - in den Vordergrund $\mathrm{zu}$ rücken (geht es etwa lediglich darum, in den Ländern des Südens Konsumenten für den elektronischen Handel und für die aus dem Norden stammenden Produkte zu gewinnen?).

Das eigentliche Problem ist nicht die digitale, sondern die soziale Kluft. Die statistisch belegten Unterschiede zwischen den Ländern des Südens und des Nordens in Bezug auf den Zugang zu den IKT sind ein Beweis für diesen sozioökonomischen Bruch (der übrigens auch innerhalb der Industrieländer feststellbar ist).

Zwar stellen die IKT unter bestimmten Voraussetzungen eine ausserordentliche Chance zur Überwindung der sozialen Kluft dar, aber nichts beweist, dass sich diese Chance nur verwirklichen lässt, wenn der Internet-Zugang für alle Menschen gewährleistet ist. Die Hindernisse, die überwunden werden müssen, damit die Menschen ihr soziales und wirtschaftliches Umfeld mit Hilfe der IKT verändern können, lassen sich nicht auf das Vorhandensein einer bezahlbaren Telekommunikationsinfrastruktur reduzieren, auch wenn diese zweifellos erforderlich ist. Es gilt noch andere Hürden zu bewältigen, etwa die Sprachbarrieren und die Bildung im Allgemeinen, und vor allen Dingen das Beherrschen der Informationskultur.

Diese Informationskultur (oder Netzwerkkultur), aber auch das Know-how hinsichtlich der Nutzung der IKT und das damit einhergehende Bewusstsein für gesellschaftliche, wirtschaftliche, sprachliche, kulturelle und ökologische (im Sinne der Informationsökologie) Herausforderungen lassen sich nur über eine umfassende Bildung und über die Praxis innerhalb der einzelnen Gruppen aneignen. Es geht darum, aus Nutzern Produzenten von Inhalten und - in ihrem unmittelbaren sozialen Umfeld - Akteure der Entwicklung zu machen, und nicht nur mehr oder weniger kritische Konsumenten. Wird darunter nicht mehr als ein kostengünstiger (oder kostenloser) Zugang und eine Schulung hinsichtlich der Nutzung lizenzierter Software auf einem PC verstanden, so profitie-

* Präsident von FUNREDES (Fundacíon Redes y Desarrollo/Networks and Development Association), Santo Domingo.

Dieser Beitrag gründet auf Überlegungen des Bürgernetzwerks MISTICA(Methodology and Social Impact of ICT in America: <www.funredes.org/mistica >. 
ren davon höchstens die Umsätze der Softwarehersteller, und es werden künftige Käufer von über den elektronischen Handel vertriebenen Produkten aus den Industrieländern herangebildet.

Zwei aus dem Süden stammende Personen mögen auf den ersten Blick vor einem Computer ein sehr ähnliches Verhalten zeigen, aber die Wirkung ihres Handelns in Bezug auf die Entwicklung kann enorm variieren. Folgende extreme Beispiele veranschaulichen dies :

$\sqsupset$ „A“ (höchstwahrscheinlich ein Yuppie aus einem Entwicklungsland) surft auf dem Netz, knüpft „Chat-Bekanntschaften“" und kauft per Internet Produkte ein, die er sich von Miami aus nach Hause liefern lässt.

$\square$ „B“ gestaltet eine Website, die seine Kultur und Sprache widerspiegelt, nimmt an berufsbezogenen E-Konferenzen teil oder arbeitet über das Netz mit anderen Kollegen zusammen, um zur Entwicklung seines Landes beizutragen; er weiss, wie er die dazu erforderlichen Informationen findet, moderiert eine virtuelle Gemeinschaft und fördert mit Hilfe seiner auf freier Software basierenden Website den Verkauf von Kunsthandwerk aus seiner Heimat.

„A“ sitzt vor einem Bildschirm, „B“ an einer Tastatur - ein kleiner, aber entscheidender Unterschied!

Wie wirkt sich das Verhalten von „A“ beziehungsweise von „B“ auf die Entwicklung ihres jeweiligen Landes aus? Im Falle von „A“ wohl eher negativ (und sogar eindeutig negativ was die Zahlungsbilanz in Dollar angeht!), hingegen zweifellos positiv im Falle von „, $\mathrm{B}^{\prime \prime}$ (und wahrscheinlich auch positiv in wirtschaftlicher Hinsicht).

Wo liegt der Unterschied zwischen dem gemeinschaftlichen Telecenter „,X“ und dem Internetcafé ,Y“? Beide bieten auf den ersten Blick vergleichbare, von mehreren Personen genutzte Ressourcen, die damit auf eine eigene Zugangsinfrastruktur (PC, Modem, Telefonanschluss) verzichten können. Der Unterschied wird erst bei näherem Hinsehen deutlich: Das Telecenter ist in ein unmittelbares soziales Umfeld eingebettet und deckt mit Hilfe von IKT verschiedene Bedürfnisse der Gemeinschaft $a b$ (zum Beispiel Rechtsberatung, Entgegennahme von Geldüberweisungen von im amerikanischen Exil lebenden Verwandten). Zudem bietet es seinen Mitgliedern Schulungsmöglichkeiten an, die auf das Empowerment abzielen. Demgegenüber ist das Internetcafé nicht in die Gemeinschaft integriert, sondern ein Dienstleistungsunternehmen, das die Kunden in die Nutzung der Software einweist und in erster Linie Unterhaltung bieten will.

Natürlich gibt es in der Praxis ein Kontinuum zwischen ,A“ und „B“ beziehungsweise zwischen „, $\mathrm{X}^{\text {“ }}$ und „Y“", und eine Schematisierung mag zu stark vereinfachen. Aber fragen wir uns ehrlich: Kann es das Ziel sein, Internet-Nutzer vom Typ „A“ auszubilden und die Errichtung von Zentren des Typs ,Y“ zu fördern?

Dies läge wohl kaum im Interesse der Zivilgesellschaft in den Entwicklungsländern. Aber genau diese 
Gefahr birgt der vereinfachende Diskurs über die ,digitale Kluft“"

Die Zivilgesellschaft in den Ländern des Südens ist beunruhigt darüber, auf welche Weise die Industrienationen und die internationalen Organisationen Aufmerksamkeit und Ressourcen für den Kampf gegen die „digitale Kluft" mobilisieren: $\mathrm{Zu}$ häufig werden als Antworten auf dieses Problem Lösungen propagiert, in denen die lokalen Akteure nicht wirklich eingebunden sind. Zudem bewirken die Finanzierungsmechanismen in den meisten Fällen, dass der Löwenanteil der Investitionen den Akteuren aus dem Norden zugute kommt (ein Beispiel unter vielen ist das von der Weltbank initiierte Projekt „Global Development Gateway“, das allein soviel kostet wie mehrere Tausend regionaler Entwicklungsprojekte wie Mistica).

Vielleicht wäre es an der Zeit, die Regeln der internationalen Zusammenarbeit zu überarbeiten, damit Initiativen der Basis vermehrt unterstuitzt werden: An die Stelle der künstlichen, weitab vom Ort des Geschehens entwickelten Lösungen, die auf der Grundlage einer absurden ökonomischen Gleichung umgesetzt werden, müssen lokale und realitätsnahe Projekte treten. Betrifft der vielfach beschworene Paradigmenwechsel, der die Herausbildung der neuen Informationsgesellschaft ermöglichen soll, nicht in erster Linie die Funktionsweise der internationalen Zusammenarbeit?
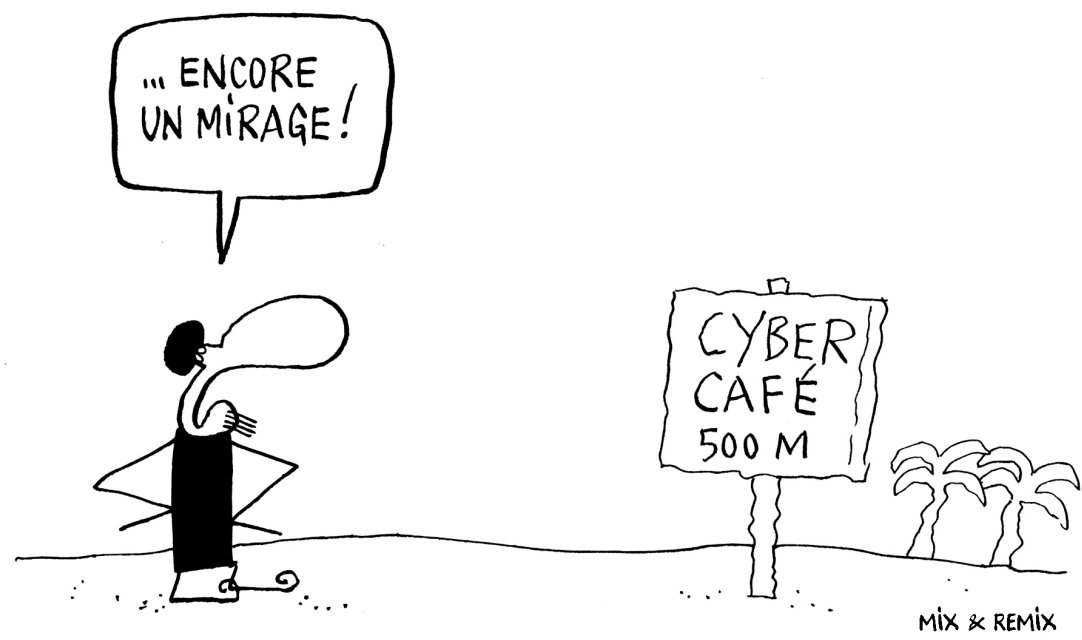

... schon wieder eine Fata Morgana! 